\title{
LA DESCENTRALIZACIÓN EN EL PERÚ
}

Situación actual, perspectivas y algunas interrogantes de fondo

\section{Carlos Monge Salgado}

F-

EL 28 DE JULIO se cumplieron tres años desde que el presidente Toledo anunciase que la descentralización sería una de las políticas centrales de su gobierno y que en noviembre del 2002 -en paralelo con las elecciones municipales programadas para esa fecha- se llevarían a cabo las elecciones para las nuevas autoridades regionales.

Desde ese momento a la fecha, el proceso descentralista ha completado su marco normativo general y el relativo a sus procesos centrales (la conformación de regiones, la descentralización fiscal y la transferencia de competencias). En estos años se han aprobado también dos planes anuales de transferencias (2003 y 2004), que son la concreción del proceso en términos de transferencias desde el Gobierno central a las regiones y las municipalidades.

\section{EL MARCO NORMATIVO}

Entre el anuncio presidencial de julio del 2001 y la elección e instalación de las nuevas autoridades regionales entre fines del 2002 e inicios del 2003, el Congreso de la República modificó los capítulos 
pertinentes de la Constitución y elaboró y aprobó la Ley de Bases de la Descentralización, la Ley de Demarcación Territorial y las leyes orgánicas de los gobiernos regionales y de las municipalidades.

De esta manera, se completó el marco normativo general de la descentralización: un mandato constitucional, una hoja de ruta, un punto de partida y un diseño para los nuevos gobiernos subnacionales.

Vale la pena resaltar algunos de los principios básicos que inspiran este marco normativo básico de la descentralización.

- La gradualidad, que supone que la descentralización es un proceso de mediano y largo plazo y no una medida o acción de corto plazo.

- La subsidiaridad, que supone que debe transferirse a los gobiernos locales y regionales todo aquello que sea materia de ser transferido para acercar las decisiones y la prestación de los servicios lo más posible a la población.

- La condicionalidad, que supone que todas las transferencias estarán supeditadas a la demostración de las capacidades de gestión de quienes aspiran a recibirlas.

- La participación y la concertación, que suponen que las decisiones cruciales de los gobiernos subnacionales sobre los recursos y el uso de las competencias transferidas se harán en consulta con los representantes de los ciudadanos.

Durante el primer semestre del año 2004 se ha aprobado el marco normativo de los tres procesos sustantivos de la descentralización. Estos tres procesos son: a) la integración para la conformación de regiones, b) la descentralización fiscal, y c) la transferencia de competencias. Las normas respectivas son la Ley de incentivos para integración y conformación de regiones, la Ley de descentralización fiscal y la Ley del sistema de acreditación.

Finalmente, en estos dos años se han aprobado los respectivos planes anuales de transferencias, en los que se concreta el proceso de transferencias de cada año. El Plan de Transferencias 2003 se centró en la transferencia de algunos proyectos especiales del Instituto Nacional de Desarrollo (INADE), de algunos programas de 
PRONAA y FONCODES, los programas de ORDESUR y algunos proyectos de Pro Vías Rural. Por su parte, el Plan Anual de Transferencias 2004 incluye ya la transferencia de algunas competencias en materia productiva, pero avanza muy poco en la transferencia de proyectos especiales de INADE y casi nada en materia de programas sociales y activos, proyectos y empresas, ahora en manos del Gobierno nacional.

\section{LOS PROCESOS EN CURSO}

Como se ha señalado antes, las normas aprobadas en el primer semestre de este año 2004 establecen los procedimientos y los plazos para el desarrollo de los tres procesos centrales de la descentralización: la conformación de regiones a partir de la integración de los actuales departamentos; la descentralización fiscal o transferencia de recursos a las regiones y localidades; y la transferencia de competencias a esas mismas regiones y localidades. Veamos cada uno de estos procesos a la luz de sus normas.

\section{La integración y conformación de regiones}

La descentralización se ha iniciado tomando como punto de partida los actuales departamentos (26, tomando como unidad política diferenciada Lima metropolitana, el Callao y las provincias de Lima). Esta opción se tomó para evitar los eventuales o inevitables conflictos en torno a qué regiones hayan de integrarse y cuáles dominasen el inicio del proceso, como ocurrió al inicio de la anterior experiencia descentralista.

La Ley de incentivos para la integración y conformación de regiones, aprobada en julio de este año, establece que pueden solicitar la integración de dos o más regiones las autoridades de esas regiones, los partidos nacionales o movimientos regionales que ahí están y los ciudadanos que en ellas habitan. Establece también que no basta con la voluntad de esas autoridades, partidos, movimientos o ciudadanos, sino que las propuestas de integración deben ser sustentadas mediante la presentación de expedientes técnicos. 


\section{CARLOS MONGE SALGADO}

Establece también la Ley que, aprobado el respectivo expediente, es finalmente la ciudadanía de las regiones en cuestión -mediante un referéndum ratificatorio- la que aprueba o desaprueba la propuesta. En el camino, se estimula -mediante una serie de incentivos- la conformación de juntas de coordinación interregional, cuyos objetivos pueden ser la gestión de proyectos de interés de más de una región o la propia integración regional. Finalmente, la ley en cuestión establece un cronograma para la realización de estas consultas: 2005, 2009, 2013 y así sucesivamente.

Hasta antes de la aprobación de la Ley de incentivos para la integración y conformación de regiones se habían constituido tres juntas de coordinación, dos de las cuales eran para gestionar proyectos de integración vial (corredores bioceánicos del norte y del sur) y una tercera en el sur para promover un proyecto de riego (Proyecto Lomas Verdes).

A partir de la aprobación de la Ley se ha intensificado el proceso de constitución de juntas y de coordinaciones en torno a posibles integraciones entre los actuales departamentos/regiones.

Al momento de redactar estas notas, las autoridades regionales de Amazonas, Cajamarca, Lambayeque, Piura, San Martín y Tumbes constituían una Junta de Coordinación Intergubernamental, con la finalidad de impulsar una cartera de proyectos de dimensión regional. En ese marco, Amazonas, Cajamarca y Lambayeque anunciaron que se proponen integrarse en una sola región y que para ello buscan cumplir con los requisitos establecidos en los plazos senalados por la ley de manera tal que se pueda lograr que en octubre del 2005 tenga lugar un referéndum ratificatorio y en noviembre del 2006 la elección de las autoridades de la nueva región.

De manera paralela, las autoridades de Pasco, Junín y Huánuco, así como las de Tacna, Moquegua, Arequipa, Puno y Madre de Dios, anunciaron que en las dos últimas semanas del mes de agosto sostendrían reuniones con objetivos similares: establecer juntas de coordinación interregional y explorar la posibilidad de procesos de integración. Lo mismo está ocurriendo en otras zonas del país.

En la agenda inmediata de este proceso se encuentra la aprobación por el CND del reglamento de la ley, que debe precisar las 


\section{LA DESCENTRALIZACIÓN EN EL PERÚ}

características de los expedientes técnicos que deben ser presentados. De la misma manera, está en agenda la propuesta de las regiones -asumida por el CND- de buscar una flexibilización de los plazos para la presentación de los expedientes técnicos y la organización de los referéndums, de manera tal que aquellas regiones que tengan voluntad de integrarse no tengan que esperar hasta el 2009 para tener la oportunidad de una consulta ratificatoria.

De la misma manera, habrá que ver cómo se materializan los incentivos a la constitución de juntas establecidos en la Ley (el acceso a recursos para el desarrollo de la cartera de proyectos de la Junta, así como prioridad en el acceso a recursos de COFIDE, del FIDE y de endeudamiento externo) y la promesa presidencial de asignar una parte proporcional de un punto del IGV (aproximadamente 820 millones de soles) a las regiones que en el referéndum del 2005 manifiesten voluntad de integración.

Ahora bien, más allá de plazos y procedimientos, el proceso anunciado desde el norte y oriente y desde el centro y el sur plantea interrogantes de fondo sobre el nuevo mapa del Perú que se podría estar configurando.

Para comenzar, ¿para qué impulsar la conformación de regiones basándose en la integración de los actuales departamentos? ¿Por qué no mantener los actuales departamentos con sus autoridades electas? La respuesta instrumental es que la sola conformación de las juntas da derecho a acceder a los incentivos antes mencionados, y la integración dará derecho a retener -por la descentralización fiscal- $50 \%$ de los recursos generados por concepto de IGV, ISC e IRPN en la nueva región.

¿Pero se trata tan sólo de acceder a más recursos? La respuesta es, con toda claridad, no. Se trata también de acceder a la posibilidad de planear el desarrollo, aplicar recursos públicos y promover la inversión privada en espacios, en territorios más amplios que los actuales departamentos. Por ejemplo, en el caso del norte y oriente, se han identificado al menos tres grandes proyectos (corredor bioceánico, reforestación de las cuencas altas y circuito turístico) que largamente desbordan el ámbito de los actuales departamentos y reclaman una visión y un manejo regional en el sentido más amplio de la palabra. 
A su vez, visiones y manejos o gestiones regionales reclaman de liderazgos regionales que superen el localismo y una visión solamente reivindicativa de las relaciones con el Gobierno nacional, y que sean capaces de articular la acción pública con la privada para generar empleos e ingresos dignos y sostenibles en los nuevos espacios regionales que se irán configurando. La integración en nuevas y amplias regiones plantea, pues, grandes retos a los partidos políticos nacionales tanto como a los movimientos políticos regionales (departamentales en la mayoría de los casos) ahora existentes. ¿Podrán agruparse esos actuales movimientos en organizaciones realmente regionales? ¿Surgirán otros? ¿Podrán los partidos nacionales desarrollar liderazgos regionales en esta nueva escala? ¿Serán capaces los partidos nacionales de contener estos liderazgos regionales tan fuertes?

\section{La descentralización fiscal y otros mecanismos de transferencia de recursos a los gobiernos regionales y locales}

A inicios de este año, al amparo de la delegación de facultades para legislar en materia tributaria, el Poder Ejecutivo aprobó la Ley de descentralización fiscal. Esta ley establece que a las regiones que culminen procesos de integración se les asignará de manera automática el $50 \%$ del IGV, el ISC y el IRPN que se generen en sus ámbitos. Se estima que esta asignación podría, en muchos casos, cuadruplicar los recursos de inversión que ahora tienen las regiones.

Al respecto, se han generado dos debates inmediatos: la metodología de estimación del aporte tributario de las regiones y la posibilidad de dar incentivos fiscales parciales a las regiones que den pasos concretos hacia la integración (como, por ejemplo, la constitución de juntas).

Sobre lo primero, en la actualidad se contabiliza la tributación ahí donde las empresas o las personas declaran su domicilio. En la medida en que la mayor parte de las grandes empresas declaran domicilio fiscal en Lima, la tributación asignada a Lima supera el $80 \%$ de la tributación nacional. Pero si se estima la tributación ahí donde se realiza la actividad económica que genera el tributo, el 
aporte de Lima a la tributación baja a menos de $50 \%$ del total, subiendo el de las regiones de manera proporcional. Es claro que con la primera metodología, el valor del $50 \%$ de los tributos que deben ser retenidos es bastante menor que en el segundo caso.

Sobre lo segundo, existe el riesgo de que un número de regiones que sí tienen voluntad de integración no logren presentar para fin de enero (plazo establecido por la ley) los respectivos expedientes y que -en consecuencia- no logren llegar a un referéndum en octubre de 2005. De ocurrir esto, tendrían que esperar hasta el año 2009 para tentar esta posibilidad. Frente a esta situación, en caso de no lograrse la reclamada flexibilización de dicho calendario, se ha planteado la posibilidad de otorgar los incentivos fiscales de manera parcial (por ejemplo, $20 \%$ de los tributos generados) a aquellas regiones que manifiesten voluntad de integrarse antes de esa fecha (por ejemplo, regiones que presenten y aprueben su expediente técnico).

Ahora bien, más allá de cuándo se haga realidad, la descentralización fiscal es un mecanismo que premia a las regiones que aportan más a la producción y la tributación, pero se han creado dos mecanismos complementarios.

El primero es el Fondo de Compensación Regional (FONCOR) que, como el FONCOMUN municipal, es un mecanismo de compensación para las regiones de menos desarrollo relativo. El MEF ya ha establecido los índices de distribución del FONCOR, pero el problema actual es su carencia de recursos, pues depende de que le asignen el $30 \%$ de los recursos que se generen por concesiones o privatizaciones. Esto lo hace un fondo impredecible y ha llevado a plantear la conveniencia de asignarle un porcentaje del IGV como mecanismo de financiamiento.

El segundo es el Fondo Intergubernamental para el Desarrollo (FIDE), fondo concursable que busca premiar la capacidad de las regiones y municipalidades de presentar proyectos que contribuyan a la integración vertical, horizontal y con el capital privado. Este fondo enfrenta el mismo problema que el FONCOR en cuanto a financiamiento, y se ha propuesto al respecto la misma solución.

Se trata, pues, de un interesante conjunto de mecanismos que premian el mayor desarrollo, compensan a las más pobres y 
premian la creatividad. Ahora bien, un problema de fondo que la puesta en práctica de esos mecanismos plantea es el impacto que esta masiva reasignación de recursos puede ejercer sobre la capacidad de acción del Gobierno central. ¿Qué cosas que ahora hace el Gobierno central tendría que dejar de hacer o traspasar a las regiones cuando se les transfieran esos recursos?

Un segundo problema que se plantea al debate es el de las capacidades actuales de los gobiernos regionales y locales de usar los recursos que ya se les vienen transfiriendo, y la capacidad que tendrían de gerenciar cantidades sustantivamente mayores. Se ha argumentado que las autoridades regionales no demuestran tener capacidad de gasto, ni siquiera de los magros recursos de que ahora disponen, y que -por tanto- no tiene sentido asignarles más. Por su parte, las regiones apuntan a la mala calidad de las carteras de proyectos heredados, a las complejidades del SNIP y a la dificultad de acceder a recursos humanos más calificados como las fuentes de estos problemas.

\section{La transferencia de competencias}

Como se ha señalado, el Plan Anual de Transferencias del 2004 ya establece la transferencia de algunas competencias de índole productiva (agro, agroindustria, pesca, minería, industria, artesanía, comercio, etc.) a las regiones y a las municipalidades.

Por su parte, la recientemente aprobada Ley del sistema nacional de acreditación establece los mecanismos para la formulación de los planes anuales de transferencia de los años que vienen, las capacidades que las regiones y las municipalidades deben acreditar para acceder a la transferencia de competencias, la necesidad de la acreditación permanente de la calidad de la gestión de las competencias transferidas y la posibilidad de que el CND tercerice la acreditación.

Respecto a la manera de organizar los planes anuales de transferencias, la ley establece que cada año el CND debe articular la oferta de los sectores del Gobierno central con la demanda de las regiones. No queda claro qué ocurrirá en el caso de que no coincidan unas con otras. 


\section{LA DESCENTRALIZACIÓN EN EL PERÚ}

Y no queda clara la imagen del país a mediano plazo, con un Gobierno central normativo en aquellas regiones y para aquellos sectores en los que se hayan dado transferencias, pero que seguirá siendo ejecutivo en aquellas regiones y para aquellas competencias no transferidas.

De la misma manera, cabe preguntarse por el sentido de la acreditación permanente. En principio, resulta necesario -como para todo el sector público- que se ponga en práctica un sistema de monitoreo de la calidad de la gestión. Pero en este caso -como en el caso de los programas sociales- no queda claro el significado y las implicaciones de este monitoreo. ¿Qué impacto tendrán los resultados de este monitoreo en el proceso de descentralización y en las autoridades que a él se someten? ¿Cuál es el estímulo y cuál la sanción? ¿Cuál es la relación que se establece de manera permanente entre los diferentes niveles de gobierno? ¿Cuáles son los márgenes de autonomía?

\section{LA PARTICIPACIÓN DE LA SOCIEDAD CIVIL}

Como se señaló al inicio de este artículo, uno de los principios normativos de la descentralización es la inclusión en los niveles subnacionales de gobierno de instancias y mecanismos para la concertación entre las autoridades electas y la sociedad civil en torno a los planes de desarrollo y los presupuestos anuales.

Estos principios se han traducido en el establecimiento de los Consejos de Coordinación Regional y Local en los Gobiernos Regionales y Locales, respectivamente. Y se han traducido también en las normas (Ley marco, reglamento e instructivos) de los presupuestos participativos regionales y locales.

La experiencia de la puesta en práctica de estos mecanismos dice que, en primer lugar, han forzado a que un conjunto de autoridades sin trayectoria participativa se vean obligadas a lidiar con el tema. Puede ser que su falta de voluntad política y las limitaciones de las normas hagan que esta participación y concertación sean nada más que una formalidad. De hecho, en muchos casos los CCR se han instalado, pero no han sesionado. $\mathrm{O}$ lo han hecho de manera tan limitada que es imposible referirse a ese funcionamiento como 
un proceso de concertación. Y no se ha completado aún, ni mucho menos, el proceso de reconformación de los CCL provinciales $y$ distritales.

En muchos otros casos, el que los CCR y CCL sean sólo consultivos (no vinculantes) y que limiten la participación de la sociedad civil al $40 \%$ de los integrantes, los convierte en una camisa de fuerza para experiencias bastante más desarrolladas de participación y concertación. Este es el caso de varias de las municipalidades que hacen parte de la Red de Municipalidades Rurales del Perú, que han desarrollado experiencias de concertación a partir de la participación de todas las organizaciones sociales de base y de representaciones territoriales, y que han dado carácter vinculante a los acuerdos que se toman en esas instancias.

Pero también es verdad que al menos colocan el tema en las agendas regionales y locales y le dan legitimidad ante autoridades poco participativas. De hecho, en muchos casos las normas sobre CCL y CCR y presupuestos participativos han permitido que actores de la sociedad civil se sientan respaldados en sus reclamos de una mayor transparencia y concertación en la gestión de los gobiernos locales y regionales.

Ahora bien, la experiencia también nos habla de limitaciones en la capacidad de la propia sociedad civil de utilizar las posibilidades que estos mecanismos brindan. En efecto, en muchos casos la desinformación, la desorganización y la falta de interés o priorización de agendas sectoriales han inhibido una mejor participación de la sociedad organizada en estas instancias y procesos.

Y cuando esta participación se ha dado, es posible observar en la propia sociedad civil la reproducción de fenómenos de exclusión. Participan más los varones urbanos de clase media y menos los pobres, los rurales, las mujeres, los indígenas.

Se hace necesario, pues, en este terreno, un triple esfuerzo de difusión de la existencia e importancia de estos mecanismos, de cabildeo para lograr la modificación de las normas en cuanto éstas limitan la participación y de superación de las formas de exclusión que se reproducen en el seno de la propia sociedad civil.

Mirando las cosas en perspectiva, y comparando la experiencia peruana con, por ejemplo, las de Brasil y Bolivia, cabe pregun- 


\section{LA DESCENTRALIZACIÓN EN EL PERÚ}

tarse por cuál será el impacto de mediano y largo plazo de la creación de la normatividad sobre la participación. Como se sabe, Bolivia optó por establecer en 1994 una legislación (la Ley de participación popular) que norma los procesos de participación y de vigilancia desde las llamadas organizaciones territoriales de base. Por su parte, la experiencia brasileña ha sido la de no regular estos procesos desde el Gobierno central, dejando más bien que la definición de cómo procesar la participación dependa de las voluntades y correlaciones políticas locales y regionales.

En el Perú, la experiencia de los 80 y 90 fue semejante a la de Brasil, en el sentido de que las experiencias de gestión participativa dependieron siempre del juego de las voluntades políticas locales y no de una legislación nacional. Sin embargo, la generación de normas, procesos y mecanismos participativos desde el Gobierno de Transición a la fecha nos coloca en una situación más parecida a la boliviana.

Habrá que ver cuál es el impacto de esta nueva orientación. ¿Se extenderá y profundizará la participación al amparo de normas nacionales que la promueven, que la hacen obligatoria? ¿Perderá vitalidad y se hará rutinaria y burocrática la participación al depender más de las normas que de las voluntades?

\section{UNA REFLEXIÓN FINAL}

La descentralización parece ser una de las pocas promesas de la transición democrática que se vienen haciendo realidad. Ciertamente, es un proceso plagado de problemas, como resultado de la carencia de un consenso programático entre sus actores que dé una perspectiva de mediano y largo plazo a los pasos que se dan en cada oportunidad. Y, sin embargo, se mueve. Lentamente a veces, sin claridad en otras, pero se mueve. Frente a la parálisis de otras reformas sustantivas del Estado (como las del Poder Judicial, de las Fuerzas Armadas y las Fuerzas Policiales, por mencionar solo tres) y la continuidad del manejo macroeconómico, la descentralización sí aparece como un proceso en curso que puede dejarnos cambios de fondo en cuanto a la distribución de recursos y de poder entre los ámbitos nacionales, regionales y locales de gobierno, y-sobre todoen las relaciones entre los ciudadanos y sus autoridades. 